Mots. Les langages du politique

$94 \mid 2010$

Trente ans d'étude des langages du politique

(1980-2010)

\title{
La caricature politique
}

\section{Marc Bonhomme}

\section{(2) OpenEdition Journals}

Édition électronique

URL : https://journals.openedition.org/mots/19858

DOI : 10.4000/mots. 19858

ISSN : 1960-6001

Éditeur

ENS Éditions

\section{Édition imprimée}

Date de publication : 30 novembre 2010

Pagination : 39-45

ISBN : 978-2-84788-235-3

ISSN : 0243-6450

Référence électronique

Marc Bonhomme, "La caricature politique », Mots. Les langages du politique [En ligne], 94 | 2010, mis en ligne le 06 novembre 2012, consulté le 28 avril 2022. URL : http://journals.openedition.org/mots/ 19858 ; DOI : https://doi.org/10.4000/mots.19858 


\section{Mots \\ Les langages du politique}

№ 94 novembre 2010

\section{Trente ans d'étude des langages du politique (1980-2010)}

ouvrage coordonné par Paul BACOT, Marlène Coulomb-GulLY, Jean-Paul Honoré, Christian Le BART, Claire Oger, Christian Plantin

\section{SOMMAIRE}

Paul Bacot, Marlène Coulomb-Gully, Jean-Paul Honoré, Christian Le Bart, Claire Oger, Christian Plantin

Le discours politique n'est pas transparent. Permanence

et transformations d'un objet de recherche

OUTILS ET ENJEUX DU DISCOURS POLITIQUE

Ruth Amossy, Roselyne Koren

Argumentation et discours politique

Christian Plantin

Argumentation-rhétorique. Les eaux mêlées

Caroline Ollivier-Yaniv

Discours politiques, propagande, communication, manipulation 
Marc Bonhomme

La caricature politique

Paul Bacot

Développement et diversification d'une onomastique politique

Ruth Wodak

The Discursive Construction of History. Brief Considerations

Henri Boyer

Les politiques linguistiques

\section{LIEUX DE LA PRODUCTION DU DISCOURS POLITIQUE}

Christian Le Bart

Parler en politique

Dominique Maingueneau

Le discours politique et son «environnement»

Alice Krieg-Planque, Claire Oger

Discours institutionnels. Perspectives pour les sciences

de la communication

Sophie Béroud, Josette Lefèvre

Le corpus syndical. Une expérience au long cours

Corinne Gobin, Jean-Claude Deroubaix

L'analyse du discours des organisations internationales.

Un vaste champ encore peu exploré

François de la Bretèque

Le retour de la parole politique dans le cinéma français

Jean-Claude Soulages

Vie et mort du citoyen cathodique

Jacques Guilhaumou

Les discours de la Révolution française.

Aperçu d'ensemble d'un trajet de recherche (1980-2009) 


\section{DISCIPLINES ET CHAMPS DE RECHERCHE POUR L'ÉTUDE DES LANGAGES DU POLITIQUE}

Philippe Braud

L'apport de la science politique à l'étude des langages du politique

Claire Blandin

L'apport de l'histoire des médias à l'étude des langages du politique

Jean-François Tétu, Bernard Lamizet

Les SIC et les langages du politique

Sylvianne Rémi-Giraud

Sémantique lexicale et langages du politique.

Le paradoxe d'un mariage difficile?

Marlène Coulomb-Gully, Juliette Rennes

Genre, politique et analyse du discours.

Une tradition épistémologique française gender blind

Johannes Angermüller

Analyser le discours politique en Allemagne (1980-2010)

Érik Neveu

L'apport de Pierre Bourdieu à l'analyse du discours.

D'un cadre théorique à des recherches empiriques

Roselyne Ringoot

Questionner le discours avec Michel Foucault.

Actualisations théoriques et actualité éditoriale

\section{ENTRETIEN}

Maurice Tournier

Mots et politique, avant et autour de 1980

La revue Mots. Les langages du politique encourage l'usage des rectifications de l'orthographe proposées par le Conseil supérieur de la langue française et approuvées par l’Académie (Journal officiel, $n^{\circ}$ 100, 6 décembre 1990). 


\section{La caricature politique}

Ces trois dernières décennies, la caricature politique a été largement analysée, comme en témoignent l'émergence de groupes d'études à son sujet ${ }^{1}$ et les revues qui l'intègrent dans leur thématique ${ }^{2}$. Si on laisse de côté les travaux de vulgarisation (catalogues d'expositions, rééditions de publications satiriques anciennes, telle L'Assiette au beurre...), les recherches sur la caricature politique depuis les années quatre-vingt révèlent trois tendances inégales selon les supports utilisés.

\section{Un intérêt prédominant pour la caricature imprimée}

Les travaux de ces trente dernières années sur la caricature politique concernent avant tout le support de l'imprimé. Émanant de chercheurs issus d'horizons variés (sciences politiques, histoire, infocom...), ces travaux se signalent par leur orientation tant diachronique que synchronique.

\section{Trois perspectives d'étude}

La plupart des études observées recouvrent le champ global de l'image caricaturale qui va de l'estampe au dessin de presse. Ces études privilégient trois angles d'investigation, en relation avec les domaines disciplinaires de leurs auteurs. Des travaux ont été plus spécifiquement axés sur les formes de la caricature politique. Ainsi, se situant dans un cadre sémiotique, Nicole EveraertDesmedt (1994) exploite les apports théoriques de Greimas pour analyser le fonctionnement narratif d'un dessin de Plantu sur la guerre du Golfe. Certaines

Université de Berne

marc.bonhomme@rom.unibe.ch

1. Parmi eux, mentionnons CORHUM, fondé en 1987 [http://www.humoresques.fr/] et l'EIRIS, créée en 1992 [http://www.eiris.eu/] (Tous les sites mentionnés dans cet article ont été consultés le 16 juin 2010).

2. Entre autres, on peut citer les livraisons suivantes: Humoresques, $n^{\circ} 5$ : Humour et politique (1994); Mots. Les langages du politique, $\mathrm{n}^{\circ} 48$ : Caricatures politiques (1996); Ridiculosa, ${ }^{\circ} 4$ : Tyrannie, dictature et caricature (1997); Hermès, no 29 : Dérision, contestation (2001); Historia, $\mathrm{n}^{\circ} 651$ : La caricature. Deux siècles de dérision salutaire (2001); La Licorne, $\mathrm{n}^{\circ} 30$ : L'étranger dans l'image satirique (2005); Ridiculosa, $\mathrm{n}^{\circ}{ }_{15}$ : Caricatures et religion(s) (2008). 
études abordent la caricature politique à travers la rhétorique visuelle. C'est le cas de Franck Malin (1995) qui, comparant les caricatures de Michel Rocard par Cabu et Pétillon, en distingue les traits visuels dûs à leur «idiolecte » de ceux inhérents au « sociolecte» du genre caricatural. De même, Laurent Gervereau (2000) dégage la grammaire figurative (exagérations, détournements...) soustendant la production des images polémiques au $20^{\mathrm{e}}$ siècle, qu'elles soient anticapitalistes ou anti-franc-maçonnes. D'autres chercheurs adoptent un point de vue iconographique, à l'instar de Laurent Baridon et Martial Guédron (2006) qui mettent en lumière l'influence des allégories moyenâgeuses sur les images de la Renaissance contre la royauté.

Divers travaux, plus nombreux, ont souligné les conditionnements contextuels de la caricature politique. Le cas type en est fourni par la méthode transversale que revendiquent plusieurs articles du numéro 48 de Mots. Les langages du politique (Caricatures politiques). En particulier, Colin Seymour-Ure (1996) y montre l'effet du renouvellement des supports (comme la fragmentation des journaux) et des médias (montée des tabloïds...) sur le rétrécissement progressif des caricatures britanniques actuelles. Ou encore Christian Delporte (1996) y met en évidence la prégnance socioculturelle des stéréotypes sur les caricatures anti-allemandes entre les deux guerres mondiales. Une même méthode transversale se retrouve dans des ouvrages qui établissent une corrélation entre les facteurs externes et les structures internes de l'image politique caricaturale. Analysant les estampes satiriques contre Louis XVI, Annie Duprat (1992) les met en rapport non seulement avec les textes pamphlétaires de l'époque révolutionnaire, mais encore avec leurs répercussions sur les sentiments populaires antiroyalistes, de telles caricatures envahissant l'espace public au lieu de rester confidentielles comme par le passé. Annie Duprat (2002) élargit sa méthode croisée en évaluant les caricatures d'Henri III et de Louis XVI à travers l'éclairage des imaginaires politiques de leur temps, plus religieux au $16^{\mathrm{e}}$ siècle - avec le recours à l'Antéchrist ou aux monstres et davantage triviaux au $18 \mathrm{e}$ siècle, avec notamment les métamorphoses de Louis XVI en porc. Quant à Bertrand Tillier (1997), centrant son étude sur les caricatures politiques du corps sous la Troisième République, il les motive par leur contexte scientifique (mode de la physiognomonie) et social (libération de la presse qui autorise toutes les audaces). En fin de compte, le corps caricaturé apparaît pour Tillier comme le lieu d'un contre-pouvoir et comme la métaphore du régime républicain désacralisé.

Mais c'est la dynamique communicationnelle de la caricature politique qui a donné lieu aux recherches récentes les plus importantes. Ses conditions de production ont été éclaircies par Christian Delporte (1993) lorsqu'il se penche sur les dessins politiques en France durant la seconde guerre mondiale. Affirmant la nécessité de ne pas dissocier ces dessins de la personnalité de leurs auteurs, il s'interroge sur les dilemmes qui se posent à eux : comment travailler 
sous le régime de Vichy sans se compromettre? Peut-on dessiner dans la zone nord sans être un collaborateur de l'occupation allemande? Delporte constate encore le comportement ambigu des caricaturistes de cette époque (entre l'appât du gain et l'engagement idéologique), ainsi que l'évolution de leurvision, allant de la certitude au doute, au fur et à mesure que la victoire allemande devient moins assurée et que la Résistance s’amplifie. Bref, selon Delporte, les dessins politiques dévoilent autant la perception que les caricaturistes ont sur leur activité que l'actualité qu'ils brocardent. D'autres recherches se sont attelées aux motivations profondes des images politiques caricaturales. Récusant l'idée que celles-ci répondent à une intention contestataire traduisant la désillusion des citoyens, Rhonda Walker (2003) soutient qu'elles défendent les opinions des propriétaires des médias, tout en renforçant les pouvoirs installés. Quelques travaux ont mis l'accent sur la manipulation du public par les caricaturistes, transformés en instruments d'une propagande officielle. Dans ce sens, Fabrice d'Almeida (1995) dissèque les caractéristiques de la propagande caricaturale - foncièrement manichéenne, tout en faisant ressortir ses stratégies défensive (contrôler les sentiments nationaux) ou offensive (déstabiliser l'adversaire). Enfin, certaines recherches ont été consacrées à la réception de la caricature politique. Tantôt leurs auteurs concluent à la relativité de cette réception, à l'exemple de Nelly Feuerhahn (1992) qui examine un dessin de Plantu portant sur la guerre en Irak et devenu énigmatique du fait de la déperdition de ses repères événementiels. Tantôt ces recherches se concentrent sur les effets de la caricature politique. Effets sur le public, quand Guillaume Doizy (2009) se demande si celle-ci fait encore peur, à la vue de l'exposition de dessins de presse organisée en 2004 à l'Assemblée nationale. Effets aussi sur les cibles de la caricature. Mentionnons à ce propos un article de Carmela Lettieri (2001) traitant des réactions brutales du premier ministre italien d'Almena suite à sa caricature par Forattini. Tantôt on a analysé la réception bloquée des images politiques caricaturales en raison de la censure. Dans cette optique, Jean-Michel Renault (2006) explore avec minutie les manifestations - entre interdiction, procès et autocensure - de cette dernière depuis deux siècles, en pointant des paradoxes. Ainsi, les pires dessins antisémites n’ont pas ému la vigilance des censeurs dans les années trente.

\section{Une focalisation sur certains genres}

Ces trente dernières années, la recherche a également eu pour objet des genres plus précis, comme l'affiche caricaturale. Ses particularismes ont été affinés, principalement par rapport au dessin de presse vis-à-vis duquel elle offre une structure plus simple et une lisibilité plus immédiate, tout en émanant d'instances politiques institutionnelles (Philippe, 1980). On relève en outre des analyses comparatives détaillées, telle celle de Philippe Buton et Laurent Gervereau (1989) sur les affiches communistes et anticommunistes entre 1919 
et 1989, dont la plupart sont caricaturales. Leur ouvrage confronte les productions des deux camps, fondées sur la haine, en expliquant comment leur thématique et leur iconicité se reflètent à travers des pratiques interdiscursives. Entre autres, l'image anticommuniste du « couteau entre les dents » se trouve fréquemment détournée par dérision dans les affiches communistes pour susciter de nouvelles significations. Inversement, les affiches communistes de la colombe de la paix sont caricaturées par l'autre camp au moyen de caviardages et de travestissements systématiques. Dans un autre ouvrage, Laurent Gervereau (1996) retrace l'évolution des affiches politiques au cours du $20^{e}$ siècle, lesquelles se font caricaturales dès qu'elles deviennent polémiques. On y voit que, sous l'influence de la publicité, celles-ci délaissent peu à peu les images symboliques complexes, comme celles de l'hydre ou du singe, au profit d'un code iconique plus dépouillé reposant sur le gros plan et l'exploitation de la couleur. De surcroît, Gervereau inventorie la diversité des positionnements mis en scène par ces affiches : faire peur, manipuler, diaboliser...

Les relations entre caricature et bande dessinée ont aussi alimenté la recherche. Didier Lefort (1991) a porté son attention sur les BD politiques, dont beaucoup ont des affinités avec la caricature. Étudiant les BD d'extrême droite parues entre 1945 et 1990 dans des journaux comme Minute ou dans des revues underground, il note l'importance des déformations graphiques liées au pastiche dans ces productions ${ }^{3}$, de même que la prégnance de la figure iconique du rat noir. De plus, son ouvrage contient une réflexion sur l'humour de droite, vu comme réactif.

Différents travaux offrent des éclairages sur des genres plus marginaux, par exemple celui des couvertures caricaturales d'ouvrages, comme celle de Martial Leiter illustrant Une Suisse au-dessus de tout soupçon de Jean Ziegler et représentant la robuste Helvetia en train de donner une bombe-biberon à un enfant noir affamé (Alamir, 1999). On s'est encore intéressé à la carte postale satirique, très développée lors des controverses sur la séparation de l’Église et de l'État au début du $20^{e}$ siècle (Doizy, Lalaux, 2005). Il convient enfin de mentionner l'étude des photomontages caricaturaux produits en France durant la première guerre mondiale, dont la composition désarticulée vise à mobiliser l'opinion publique contre l'ennemi (de Pertuis, 2007).

\section{Des recherches très ciblées sur la caricature télévisuelle}

Comportant deux dimensions supplémentaires par rapport à la caricature imprimée, celles de la marionnette animée et de la mise en sketch, la carica-

3. Parmi elles, Didier Lefort cite «Le monstre de Markstein » d'A. D. E. et «La rose et le chardon » de Marty, respectivement à l'encontre de Georges Marchais et de François Mitterrand. 
ture politique télévisuelle a essentiellement été analysée depuis une quinzaine d'années à travers ses deux émissions phares : le Bébête Show et les Guignols de l'info. Certaines études brossent un tableau contrastif entre ces deux émissions satiriques. Ainsi, Marlène Coulomb-Gully (2001) estime que, si elles ont des points communs (visée moraliste et rhétorique du portrait-charge), leur univers diffère profondément. Quand le Bébête Show donne à voir un mode de caricature passéiste, basé aussi bien sur des figurines stéréotypées que sur un clivage clair entre la réalité politique et sa représentation médiatique, les Guignols de l'info témoignent d'un traitement caricatural plus moderne. Celui-ci tient à un renouvellement des procédés (recours au morphing et à des personnages très mobiles), à un humour davantage implicite ainsi qu'à une confusion entre le fait politique et sa médiatisation.

D'autres recherches ont mis en avant des aspects spécifiques à ces deux émissions caricaturales. À propos du Bébête Show, on s'est attaché aux modalités de sa diffusion médiatique. Annie Collovald (1992) examine les motivations de ses producteurs. Selon elle, loin d'avoir une intention seulement satirique, ceux-ci ont cherché à se réapproprier un espace politique discrédité en se montrant davantage soucieux des attentes éthiques du public que les professionnels ridiculisés. Pour sa part, Grégory Derville (1996) porte ses remarques sur le contenu de cette émission, traitement lexicométrique à l'appui. Il en déduit la dimension populaire et populiste du Bébête Show, dans la mesure où celuici se moule sur l'idéologie finalement conservatrice du public. Dans un autre article (2001), Grégory Derville explicite la réception paradoxale du Bébête Show : si son anarchisme apparent semble menacer la démocratie, il est en fait largement récupéré par les instances politiques, ce qui aboutit à sa banalisation et à son institutionnalisation. Pour ce qui est des Guignols de l'info, ils ont surtout donné lieu à des investigations sur le fonctionnement singulier de leurs déformations burlesques. Annie Collovald et Érik Neveu (1996) font ressortir leur statut méta-caricatural : en représentant une représentation, à savoir un journal télévisé caricaturé, ils offrent un traitement en abîme de la vie politique, confiné au seul univers audiovisuel. Aymeric Maurice (2004) étend le débat sur l'intertextualité des Guignols. Celle-ci se construit de l'intérieur, par la réapparition du même personnel politique grotesque à travers la récurrence des émissions, mais également de l'extérieur, en raison de leurs multiples allusions médiatico-culturelles décalées.

\section{Caricature politique et Internet. Des perspectives à explorer}

Si les recherches sur la caricature politique depuis trente ans concernent majoritairement ses productions graphiques et, épisodiquement, ses réalisations télévisuelles, on constate encore peu d'études sur les relations entre 
caricature politique et Internet. À leur sujet, on ne relève guère que deux pistes de réflexion. D'un côté, les avis sont partagés sur le fait qu'Internet rénove les configurations caricaturales. Tandis que certains, comme Laurent Gervereau (2000), pensent que l'outil informatique ne modifie pas vraiment les formes de caricature, d'autres, tel Jean-Paul Gourévitch (1998), estiment qu'il favorise le développement des photomontages satiriques (à l'exemple de ceux produits contre George Bush) et l'expansion de nouvelles images caricaturales, comme les distorsions animées (cas du «Jospinocchio», caricature de Lionel Jospin dans les années deux mille, dont le nez s'allongeait au fur et à mesure de ses mensonges supposés). D'un autre côté, la plupart des observateurs sont d'accord avec l'idée qu'Internet permet une médiatisation extrême de la caricature politique, ce qui avive les tensions interculturelles. Jean-Michel Renault (2006) signale à ce propos l'affaire des caricatures danoises de Mahomet, qui n'ont dû qu'à leur diffusion par le Web le scandale politico-religieux qu'elles ont suscité sur le plan mondial.

Par-delà ces considérations, l'essor actuel de la caricature politique sur Internet est de nature à fournir des perspectives de recherche prometteuses. Celles-ci peuvent consister en l'étude des sites spécialisés dans l'image satirique 4 et en l'analyse de la grande interactivité insufflée à la communication caricaturale par les blogs et les forums commentant les dessins de l'actualité politique5. Il serait intéressant d'évaluer comment ces formes électroniques d'expression contribuent à la vitalité du débat démocratique, en introduisant des espaces de liberté vis-à-vis des pouvoirs en place.

\section{Références}

ALAMIR M., 1999, «Martial Leiter, ou quand l'humour noir voit rouge», Humoresques, $\mathrm{n}^{\circ} 10, \mathrm{p} \cdot 39-56$.

BARIDON L., GUÉdRon M., 2006, L'Art et l'histoire de la caricature, Paris, Citadelles \& Mazenod.

Buton Ph., Gervereau L., 1989, Le couteau entre les dents, Paris, Chêne.

Collovald A., 1992, "Le Bébète Show, idéologie journalistique et illusion critique», Politix, nº19, p. 67-86.

Collovald A., Neveu É., 1996, «Les Guignols ou la caricature en abîme », Mots. Les langages du politique, $\mathrm{n}^{\circ} 48$, septembre, p. 87-112.

Coulomb-Gully M., 2001, «Petite généalogie de la satire politique télévisuelle. L'exemple des Guignols de l'info et du Bébête Show», Hermès, n² 29, p. 33-42.

4. Parmi ces sites, le plus élaboré est http://www.caricaturesetcaricature.com/.

5. Pour les blogs de caricatures politiques, voir http://www.dessin-humour.com/, http://devil65. skyrock.com/ ou http://www.fond-ecran.pixelenvrac.com/. Quant aux forums sur les dessins politiques, on en trouve aux adresses suivantes : http://www.canalblog.com/, http://forum. fluctuat.net/, http://www.iconovox.com/blog/. 
D’AlmeidA F., 1995, Images et propagande, Paris, Casterman.

DeLPORTE Ch., 1993, Les crayons de la propagande. Dessinateurs et dessin politique sous l'Occupation, Paris, CNRS.

- 1996, «Méfions-nous du sourire de Germania! L’Allemagne dans la caricature française (1919-1939) », Mots. Les langages du politique, nº 48, septembre, p. 33-54.

De Perthuis B., 2007, «L'imagerie de la Grande Guerre. Vers un nouvel esthétisme du discordant, du disharmonieux et de l'aléatoire », Ridiculosa, n¹4, p. 107-128.

DeRVILLE G., 1996, "Le discours du Bébète Show de TF1 : populaire ou populiste?», Mots. Les langages du politique, $\mathrm{n}^{\circ} 48$, septembre, p. 115-126.

— 2001, «Élément de démocratie ou menace pour la démocratie? Récupération, disqualification et routinisation du Bébête Show», Hermès, n 29, p.145-154.

Doızy G. , 2009, "L'histoire du dessin de presse», Quel avenir pour le dessin de presse?, Paris, Centre Pompidou, p.5-19.

DoIzY G., LALAUX J.-B., 2005, À bas la calotte. La caricature anticléricale et la séparation des Églises et de l'État, Paris, Alternatives.

Duprat A., 1992, Le roi décapité. Essai sur les imaginaires politiques, Paris, Cerf.

- 2002, Les rois de papier. La caricature de Henri III à Louis XVI, Paris, Belin.

EVERAERT-Desmedt N., 1994, "La médiation impossible. Interprétation d’un dessin de Plantu », Degrés, n079-80, p.d-d16.

FEUERHAHN N., 1992, "L'humour des confettis. Le dessin de presse et la représentation ludique de l'information », Humoresques, n³, p. 63-77.

Gervereau L, 1996, Histoire mondiale de l'affiche politique, Paris, France Loisirs.

- 2000, Un siècle de manipulation par l'image, Paris, Somogy.

GouréVITCH J.-P., 1998, L'image en politique, Paris, Hachette.

LEFORT D., 1991, Les bandes dessinées et dessins de presse de l'extrême droite, Marseille, Bédésup.

LETTIERI C., 2001, «Le dessin de presse au tribunal. Les enseignements de la querelle d'Almena/Forattini », Hermès, nº 29, p. 155-164.

MALIN F., 1995, "Le dessin de presse a-t-il un style?», Le style, G. Maurand éd., Toulouse, Cals, p. 271-288.

MAURICE A., 2004, "Les rapports intertextuels dans la constitution des personnages des Guignols de l'info ", L'intertextualité, P. Marillaud éd., Toulouse, Cals, p. 25-39.

PHILIPPE R., 1980, Affiches et caricatures dans l'histoire, Paris, Nathan.

Renault J.-M., 2006, Censure et caricature, Montpellier, Pat à Pan.

SEYMour-URE C., 1996, "Le dessin satirique dans la presse britannique contemporaine », Mots. Les langages du politique, $\mathrm{n}^{\circ} 48$, septembre, p. 55-73.

TILLIER B., 1997, La Républicature. La caricature politique en France (1870-1914), Paris, CNRS.

WALKER R., 2003, "La caricature politique sous son vrai jour», Revue parlementaire canadienne, printemps, p.16-21. 